\title{
EchoGéo
}

$14 \mid 2010$

Afrique, 50 ans d'indépendance : Dynamiques spatiales, identités, circulations

\section{Températures et indices bioclimatiques dans le vignoble du Val de Loire dans un contexte de changement climatique}

Cyril Bonnefoy, Hervé Quenol, Olivier Planchon et Gérard Barbeau

\section{OpenEdition}

Journals

Édition électronique

URL : https://journals.openedition.org/echogeo/12146

DOI : $10.4000 /$ echogeo.12146

ISSN : 1963-1197

Éditeur

Pôle de recherche pour l'organisation et la diffusion de l'information géographique (CNRS UMR 8586)

Référence électronique

Cyril Bonnefoy, Hervé Quenol, Olivier Planchon et Gérard Barbeau, « Températures et indices

bioclimatiques dans le vignoble du Val de Loire dans un contexte de changement climatique »,

EchoGéo [En ligne], 14 | 2010, mis en ligne le 13 décembre 2010 , consulté le 31 juillet 2021. URL:

http://journals.openedition.org/echogeo/12146; DOI : https://doi.org/10.4000/echogeo.12146

Ce document a été généré automatiquement le 31 juillet 2021.

EchoGéo est mis à disposition selon les termes de la licence Creative Commons Attribution - Pas d'Utilisation Commerciale - Pas de Modification 4.0 International (CC BY-NC-ND) 


\title{
Températures et indices bioclimatiques dans le vignoble du Val de Loire dans un contexte de changement climatique
}

\author{
Cyril Bonnefoy, Hervé Quenol, Olivier Planchon et Gérard Barbeau
}

\section{Introduction}

1 La géographie des vignobles mondiaux est étroitement liée au zonage climatique. Le climat n'est certes qu'un élément composant des terroirs mais explique pour beaucoup le comportement de la vigne et les caractéristiques du vin produit (Galet, 2000). Ainsi, à cause du réchauffement climatique planétaire, cette géographie pourrait être remise en question (Vaudour, 2003 ; White et al., 2006 ; Lobell et al., 2006 ; Webb et al., 2007). C'est pourquoi, une démarche prospective concernant ces changements du climat doit être adoptée dès maintenant pour pouvoir anticiper les futurs impacts sur les vignobles. Dans ce contexte, a été créé le projet de recherche international «TERVICLIM » qui a pour objectif de mettre en place une méthodologie de mesures (météorologiques et agronomiques) et de modélisation spatiale du climat adaptée aux échelles fines. Démarche qui permettra d'une part, de définir le climat actuel des vignobles et, d'autre part, d'apporter des réponses aux conséquences futures du changement climatique. Cependant, avant de descendre aux échelles les plus fines, il est nécessaire d'avoir une connaissance approfondie du climat régional. Cet article présente les premiers résultats à l'échelle régionale pour l'une des régions cibles du programme, le Val de Loire (carte 1). Les nuances climatiques du Centre-Ouest français où se trouve notamment le Val de Loire sont donc étudiées. Un diagnostic du réchauffement climatique est réalisé à partir de séries débutant au milieu de XXe siècle. Par ailleurs, d'autres études ont déjà montré auparavant les nuances du changement climatique à l'échelle d'une région viticole et les effets déjà notables sur les vignobles (Jones et al., 2005 ; Madelin et al., 2007 ; Seguin, 2010). Une première partie présente les données utilisées pour cette étude, tandis 
qu'une seconde explique les méthodes; une dernière partie expose les résultats concernant la description des influences climatiques, du réchauffement climatique et de ses premiers impacts sur les indices bioclimatiques viticoles de la région d'étude.

Figure 1 - Carte de localisation du Val de Loire, de ces principaux vignobles et des stations MétéoFrance pour cette région

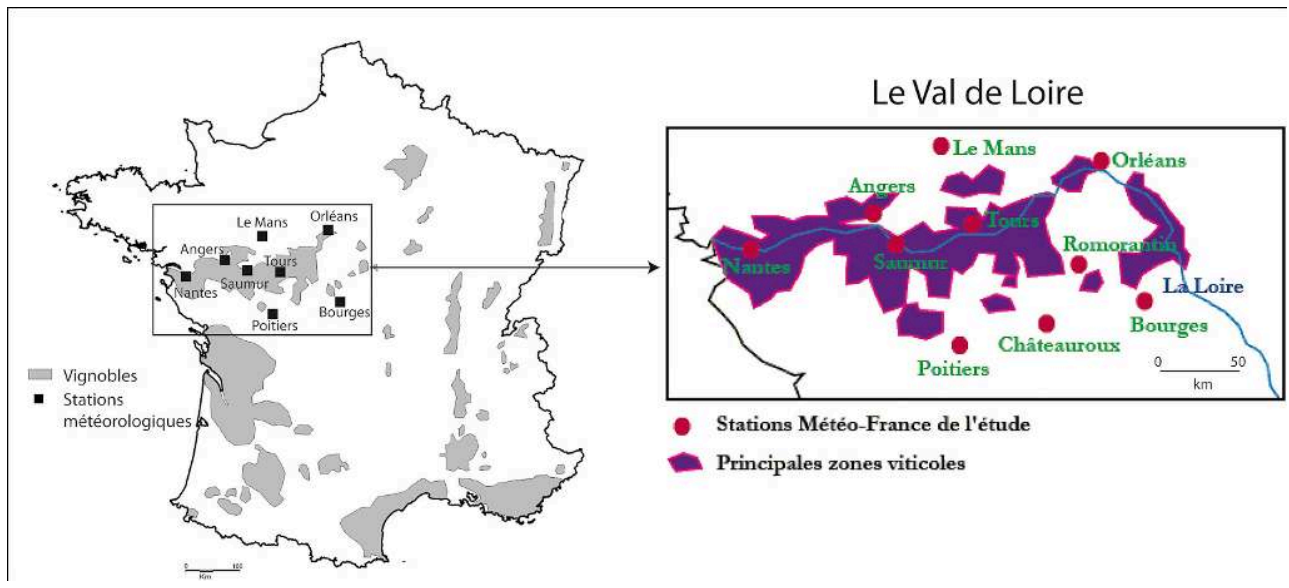

Source : d'après Quenol et al, 2008.

\section{Les données climatiques utilisées}

2 Afin d'étudier les nuances climatiques régionales, les données de dix stations du Centre-Ouest de la France ont été obtenues par la base de données de Météo - France (outil d'extraction Climathèque) et ont été analysées (tableau 1). Huit de ces stations sont situées dans le Val de Loire. Ces stations sont localisées dans les régions administratives Pays de la Loire (quatre stations) et Centre (six stations). La station actuelle de Tours (Parçay-Meslay), ouverte en 1959, a été déplacée en 1964 d'environ $2 \mathrm{~km}$ vers l'extérieur de la ville (aérodrome). Ainsi, lorsque l'on étudie la série de données, une baisse de la température apparaît au milieu des années 60 alors que cette tendance à la baisse n'apparaît pas dans les autres stations qui sont restées fixes. 
Tableau 1 - Coordonnées géographiques des stations et longueur de l'échantillon temporel disponible

\begin{tabular}{|c|c|c|c|c|c|}
\hline & Alt & Lat & Long & Situation & Données \\
\hline Nantes & $26 \mathrm{~m}$ & $47^{\circ} 09^{\prime} 00^{\prime \prime} \mathrm{N}$ & $01^{\circ} 36^{\prime} 30^{\prime \prime} \mathrm{W}$ & Aéroport - Plaine & 1946-2008 \\
\hline Angers & $50 \mathrm{~m}$ & $47^{\circ} 28^{\prime} 42^{\prime \prime} \mathrm{N}$ & $00^{\circ} 36^{\prime} 48^{\prime \prime} \mathrm{W}$ & Plaine & $1947-2008$ \\
\hline Saumur & $69 \mathrm{~m}$ & $47^{\circ} 15^{\prime} 06^{\prime \prime} \mathrm{N}$ & $00^{\circ} 04^{\prime} 24^{\prime \prime} \mathrm{W}$ & $\begin{array}{l}\text { Jardin des plantes - Plateau peu élevé } \\
\text { (environ } 30 \mathrm{~m} \text { au dessus de la Loire) }\end{array}$ & 1951-2008 \\
\hline \multirow{2}{*}{ Tours } & $96 \mathrm{~m}$ & $47^{\circ} 25^{\prime} 00^{\prime \prime} \mathrm{N}$ & $00^{\circ} 42^{\prime} 00^{\prime \prime} \mathrm{E}$ & Plateau (environ $50 \mathrm{~m}$ au dessus de la Loire) & 1946-1964 \\
\hline & $108 \mathrm{~m}$ & $47^{\circ} 26^{\prime} 36^{\prime \prime} \mathrm{N}$ & $00^{\circ} 43^{\prime} 36^{\prime \prime} \mathrm{E}$ & $\begin{array}{c}\text { Aérodrome - Plateau } \\
\text { (un peu plus de } 50 \mathrm{~m} \text { au dessus de la Loire) }\end{array}$ & $1964-2008$ \\
\hline Châteauroux & $158 \mathrm{~m}$ & $46^{\circ} 52^{\prime} 06^{\prime \prime} \mathrm{N}$ & $01^{\circ} 44^{\prime} 24^{\prime \prime} \mathrm{E}$ & Aérodrome - Plaine & 1946-2008 \\
\hline Bourges & $161 \mathrm{~m}$ & $47^{\circ} 03^{\prime} 30^{\prime \prime} \mathrm{N}$ & $02^{\circ} 21^{\prime} 30^{\prime \prime} \mathrm{E}$ & Aérodrome - Plateau & 1946-2008 \\
\hline Poitiers & $123 \mathrm{~m}$ & $46^{\circ} 35^{\prime} 36^{\prime \prime} \mathrm{N}$ & $00^{\circ} 18^{\prime} 48^{\prime \prime} \mathrm{E}$ & Aérodrome - Plateau & 1946-2008 \\
\hline Le Mans & $48 \mathrm{~m}$ & $47^{\circ} 56^{\prime} 42^{\prime \prime} \mathrm{N}$ & $00^{\circ} 11^{\prime} 36^{\prime \prime} \mathrm{E}$ & Aérodrome - Plaine & 1946-2008 \\
\hline Romorantin & $83 \mathrm{~m}$ & $47^{\circ} 19^{\prime} 06^{\prime \prime} \mathrm{N}$ & $01^{\circ} 41^{\prime} 12^{\prime \prime} \mathrm{E}$ & Aérodrome - Vallée légèrement encaissée & $1953-2008$ \\
\hline Orléans & $125 \mathrm{~m}$ & $47^{\circ} 59^{\prime} 00^{\prime \prime} \mathrm{N}$ & $01^{\circ} 46^{\prime} 36^{\prime \prime} \mathrm{E}$ & $\begin{array}{c}\text { Aérodrome - Plateau } \\
\text { (environ } 35 \mathrm{~m} \text { au dessus de la Loire) }\end{array}$ & 1946-2008 \\
\hline
\end{tabular}

Les données traitées ici sont les températures minimales, maximales et moyennes mensuelles depuis le milieu du XXe siècle (1947 pour Angers, 1951 pour Saumur, 1953 pour Romorantin et 1946 pour les autres stations) jusqu'à décembre 2008. La longueur des chroniques permet donc d'analyser l'évolution thermique sur une période suffisamment longue, et de caractériser et quantifier le réchauffement climatique dans le Val de Loire.

4 Les données journalières des stations de Nantes, Angers, Saumur et Tours ont été également utilisées pour calculer certains indices bioclimatiques. Ces données sont disponibles depuis 1950 pour Nantes, Angers et Saumur mais seulement depuis 1960 pour Tours.

\section{Les méthodes de diagnostic climatique}

5 Afin de différencier les nuances climatiques entre les différentes stations, les normales des températures moyennes mensuelles ont été calculées sur la période 1961-1990 qui est utilisée comme période de référence dans les modèles climatiques globaux et donc, pour les adaptations anticipées.

6 Plusieurs méthodes d'analyse statistique des données ont été utilisées afin de caractériser le réchauffement climatique régional et étudier l'évolution des indices bioclimatiques, qui sont de bons indicateurs pour la phénologie de la vigne.

7 La détection des ruptures climatiques a été effectuée à l'aide du test statistique de Pettitt. La stationnarité des séries temporelles de températures de toutes les stations a été étudiée afin de déterminer si une tendance significative au réchauffement peut être identifiée depuis le milieu du XXe siècle. Comme l'indique Lubes et al. (1994), "une rupture peut être définie par un changement dans la loi de probabilité de la série chronologique à un instant donné ». Le test de Pettitt est non-paramétrique et dérive du test de MannWhitney (Pettitt, 1979). Ce test permet de détecter la présence d'une rupture dans une série temporelle. L'absence de rupture dans la série est considérée comme l'hypothèse nulle d'homogénéité. 
Des indices bioclimatiques adaptés à la viticulture ont été calculés. Ces indices sont plus pertinents à utiliser que les données de températures brutes, dans le cas d'une étude de l'influence du climat sur la vigne. L'évolution de l'indice héliothermique de Huglin (Huglin et Schneider, 1998) a été étudiée pour des stations proches des vignobles (Nantes, Angers, Saumur et Tours).Cet indice permet la classification des vignobles dans différentes catégories de climats du type frais au type chaud et il peut être mis en relation avec les différents stades phénologiques (tableau 2).L'évolution de l'indice des degrés jours de Winkler (Winkler et al., 1974) a également été étudiée pour les mêmes stations notamment pour étudier la relation avec la teneur en sucre du raisin (encadré 1).

Tableau 2 - Classification des climats viticoles d'après l'indice héliothermique de Huglin

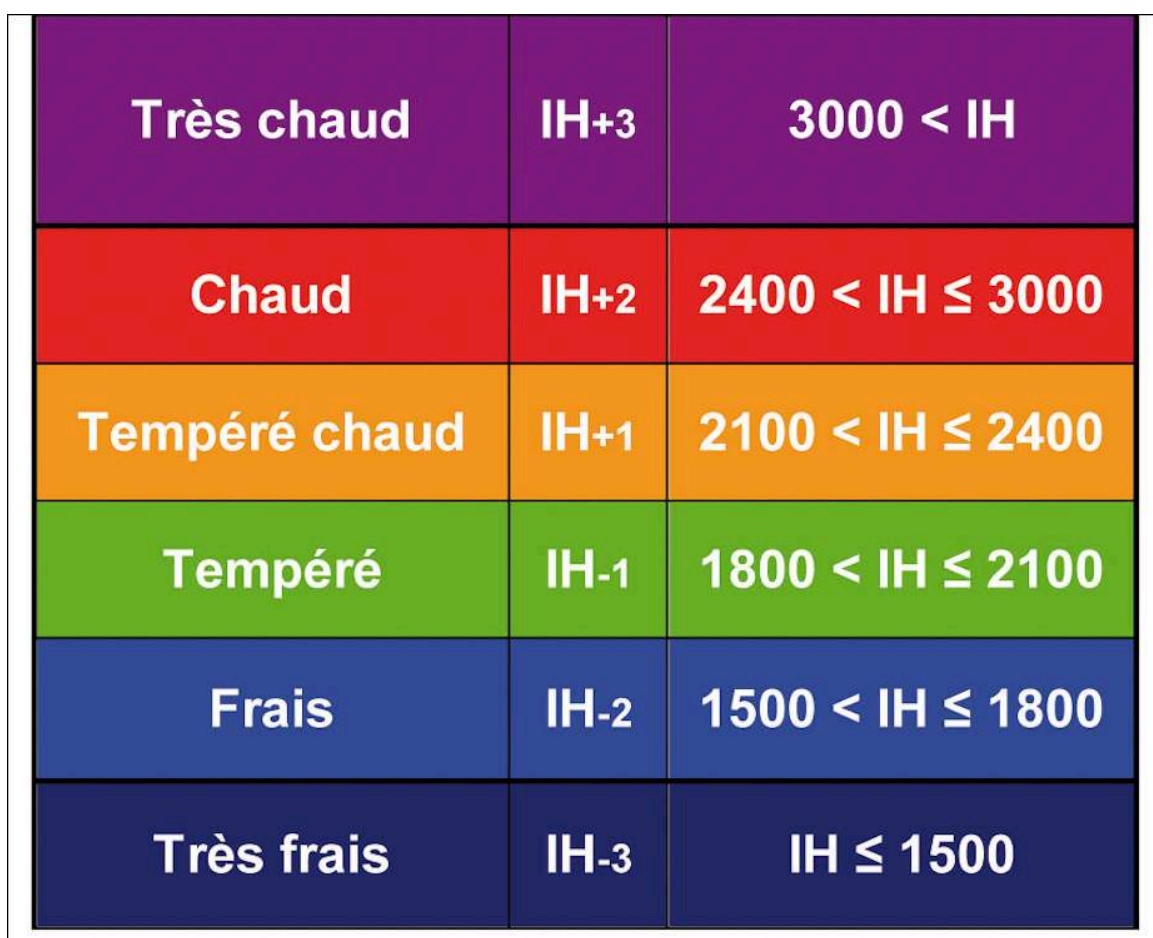

D'après Huglin et al., 1998

Encadré 1 - Indices bioclimatiques utilisés en viticulture

La valeur de l'indice de Huglin pour une station correspond à la valeur cumulée de l'indice à la date du 30 septembre, le cumul se faisant depuis le $1^{\mathrm{er}}$ avril.

Indice d'Huglin $=\Sigma[(T m-10)+(T x-10) / 2]^{*} k$

où $\mathrm{Tm}=$ Température moyenne, $\mathrm{Tx}=$ Température maximale et $\mathrm{k}$ le coefficient de longueur du jour variant de 1,02 à 1,06 entre 40 et 50 degrés de latitude.

L'indice des degrés jours de Winkler correspond à la somme des températures moyennes journalières à partir de la base de $10^{\circ} \mathrm{C}$ qui est effectuée du $1^{\mathrm{er}}$ avril au 31 octobre :

Degrés-jours $=\Sigma \mathrm{T}_{\mathrm{j}}-10\left(\right.$ avec $\left.\mathrm{T}_{\mathrm{j}}>10\right)$ 


\section{Résultats et discussion}

\section{Principaux caractères climatiques régionaux}

9 L'influence océanique marquée à Nantes, station bénéficiant du climat maritime propre à la bordure atlantique française (Planchon, 1994 et 1997), s'atténue lorsqu'on se dirige vers l'intérieur des terres (tableau 3). En effet, certains caractères climatiques « continentaux » apparaissent progressivement en direction de l'est, à travers les bas plateaux du sud du bassin parisien (Pédelaborde, 1957 ; Pagney, 1988). Ainsi, à Nantes qui représente bien le climat maritime atlantique, les hivers y sont les plus doux avec la température moyenne du mois de janvier la plus élevée de toutes les stations $\left(5,3^{\circ} \mathrm{C}\right)$ et des étés sans grande chaleur (température moyenne du mois de juillet de $19,1^{\circ} \mathrm{C}$ ). L'amplitude thermique annuelle reste ainsi relativement faible $\left(13,8^{\circ} \mathrm{C}\right)$. Cette douceur hivernale liée à la proximité de l'océan s'atténue progressivement vers l'est. Les autres stations (hormis Angers et Saumur) connaissent toutes des hivers plus rigoureux avec une température moyenne en janvier comprise entre 3,2 et $4,1^{\circ} \mathrm{C}$. L'amplitude thermique annuelle de ces mêmes stations est plus élevée en raison d'un été plutôt chaud; elle atteint par exemple près de $16^{\circ} \mathrm{C}$ à Bourges et à Châteauroux. Cependant, à l'effet de la continentalité s'ajoute l'influence de l'altitude ou, plus précisément, de la topographie. En effet, plusieurs stations de référence dans cette étude (Tours, Bourges, Poitiers et Orléans) sont situées sur des plateaux peu élevés (environ 100 à $150 \mathrm{~m}$ ), mais toutefois suffisamment surélevés par rapport aux vallées qui les traversent, y compris la Loire. Bien que située dans une vallée très peu encaissée, Romorantin ressort comme la station la plus froide en hiver (température minimale moyenne en janvier de $0,2^{\circ} \mathrm{C}$ ). Cette station est située dans une plaine sableuse, donc sur des sols qui amplifient les effets du rayonnement nocturne (Oke, 1978; Escourrou, 1981). De telles caractéristiques permettent ainsi de comparer la station de Romorantin à celle de Mont-de-Marsan dans les Landes (Avila, 1986). Dans les deux cas, l'affaiblissement du vent sur des espaces à dominante forestière (forte rugosité ; Pinty et al., 1989) aggrave les possibilités de gelées de rayonnement. A l'inverse, et pour les mêmes raisons liées au rayonnement des sols sableux, Romorantin fait partie des stations les plus chaudes en été. Enfin, la position latitudinale, même si les différences entre les stations sont moins marquées qu'en longitude, a également un effet sur les températures. Par exemple, à altitude et situation comparable, la température moyenne de janvier à Orléans est de $1^{\circ} \mathrm{C}$ plus basse que celle de Poitiers. 
Tableau 3 - Températures mensuelles dans le Centre-Ouest français pour 10 stations climatiques représentatives (normales 1961-1990)

\begin{tabular}{|c|c|c|c|c|c|c|c|c|c|c|c|c|c|c|}
\hline Station & $\begin{array}{l}\text { Temp. } \\
\left(\text { en }{ }^{\circ} \text { C) }\right.\end{array}$ & Jan & Fév & Mar & Avr & Mai & Jui & Juil & Aoû & Sep & oct & Nov & Déc & An \\
\hline \multirow{3}{*}{ Nantes } & Tmax & 8,3 & 9,6 & 12,1 & 14,8 & 18,2 & 21,8 & 24,3 & 23,9 & 21,7 & 17,3 & 12,0 & 8,9 & 16,1 \\
\hline & Tmoy & 5,3 & 6,2 & 8,0 & 10,3 & 13,5 & 16,8 & 19,1 & 18,7 & 16,7 & 13,1 & 8,5 & 6,0 & 11,9 \\
\hline & $T \min$ & 2,4 & 2,8 & 3,9 & 5,9 & 8,9 & 11,8 & 13,8 & 13,4 & 11,8 & 8,9 & 5,0 & 3,0 & 7,6 \\
\hline \multirow{3}{*}{ Angers } & $T \max$ & 7,6 & 9,0 & 11,9 & 14,8 & 18,3 & 22,0 & 24,6 & 24,2 & 21,8 & 17,0 & 11,2 & 8,1 & 15,9 \\
\hline & Tmoy & 4,7 & 5,6 & 7,7 & 10,1 & 13,4 & 16,8 & 19,0 & 18,7 & 16,5 & 12,6 & 7,8 & 5,3 & 11,5 \\
\hline & Tmin & 1,8 & 2,2 & 3,5 & 5,4 & 8,6 & 11,5 & 13,4 & 13,1 & 11,2 & 8,2 & 4,3 & 2,5 & 7,1 \\
\hline \multirow{3}{*}{ Saumur } & $T \max$ & 7,5 & 9,2 & 12,2 & 15,4 & 19,1 & 22,1 & 25,6 & 24,9 & 22,3 & 17,3 & 11,3 & 8,0 & 16,3 \\
\hline & Tmoy & 4,6 & 5,8 & 8,0 & 10,6 & 14,0 & 16,8 & 19,7 & 19,2 & 16,9 & 12,9 & 7,9 & 5,2 & 11,8 \\
\hline & $\min$ & 1,8 & 2,4 & 3,7 & 5,8 & 8,9 & 11,5 & 13,8 & 13,4 & 11,4 & 8,5 & 4,5 & 2,4 & 7,3 \\
\hline \multirow{3}{*}{ Le Mans } & Tmax & 7,0 & 8,5 & 11,5 & 14,6 & 18,3 & 21,9 & 24,4 & 23,9 & 21,4 & 16,6 & 10,7 & 7,7 & 15,6 \\
\hline & Tmoy & 4,1 & 5,0 & 7,1 & 9,7 & 13,2 & 16,6 & 18,7 & 18,3 & 15,9 & 12,1 & 7,2 & 4,8 & 11,1 \\
\hline & $T \min$ & 1,2 & 1,5 & 2,7 & 4,8 & 8,1 & 11,3 & 13,1 & 12,6 & 10,4 & 7,6 & 3,7 & 1,9 & 6,6 \\
\hline \multirow{3}{*}{ Tours } & Tmax & 6,6 & 8,1 & 11,2 & 14,4 & 18,1 & 21,7 & 24,7 & 24,3 & 21,6 & 16,6 & 10,4 & 7,1 & 15,4 \\
\hline & Tmoy & 3,9 & 5,0 & 7,2 & 9,8 & 13,2 & 16,6 & 18,9 & 18,6 & 16,3 & 12,2 & 7,2 & 4,5 & 11,1 \\
\hline & Tmin & 1,3 & 1,8 & 3,2 & 5,1 & 8,3 & 11,4 & 13,1 & 12,9 & 10,9 & 7,9 & 3,9 & 2,0 & 6,8 \\
\hline \multirow{3}{*}{ Orléans } & & 6,0 & 7,6 & 10,8 & 14,3 & 17,9 & 21,6 & 24,5 & 24,1 & 21,2 & 16,2 & 9,9 & 6,5 & 15,0 \\
\hline & Tmoy & 3,2 & 4,2 & 6,6 & 9,3 & 12,8 & 16,2 & 18,5 & 18,2 & 15,8 & 11,7 & 6,6 & 3,8 & 10,6 \\
\hline & Tmin & 0,3 & 0,8 & 2,4 & 4,3 & \begin{tabular}{|l|}
7,8 \\
\end{tabular} & 10,7 & 12,6 & 12,3 & 10,3 & 7,3 & 3,3 & 1,1 & 6,1 \\
\hline \multirow{3}{*}{ Romorantin } & $T \max$ & 6,8 & 8,8 & 11,9 & 15,2 & 18,9 & 22,5 & 25,3 & 24,7 & 22,0 & 17,0 & 10,7 & 7,4 & 15,9 \\
\hline & Tmoy & 3,5 & 4,7 & 6,8 & 9,5 & 13,1 & 16,2 & 18,4 & 17,9 & 15,4 & 11,5 & 6,6 & 4,1 & 10,6 \\
\hline & $\min$ & 0,2 & 0,6 & 1,7 & 3,8 & 7,2 & 10,0 & 11,6 & 11,1 & 8,8 & 6,1 & 2,6 & 0,8 & 5,4 \\
\hline \multirow{3}{*}{ Chateauroux } & Tmax & 6,3 & 8,2 & 11,1 & 14,4 & 18,2 & 21,7 & 25,0 & 24,2 & 21,6 & 16,7 & 10,3 & 6,9 & 15,4 \\
\hline & Tmoy & 3,4 & 4,7 & 6,9 & 9,7 & 13,2 & 16,5 & 19,1 & 18,7 & 16,3 & 12,1 & 6,9 & 4,0 & 10,9 \\
\hline & Tmin & 0,5 & 1,2 & 2,6 & 4,9 & 8,2 & 11,2 & 13,2 & 13,1 & 11,0 & 7,6 & 3,5 & 1,2 & 6,5 \\
\hline \multirow{3}{*}{ Bourges } & Tmax & 6,2 & 8,2 & 11,3 & 14,6 & 18,3 & 22,0 & 25,2 & 24,6 & 21,8 & 16,7 & 10,2 & 6,8 & 15,5 \\
\hline & Tmoy & 3,3 & 4,7 & 7,0 & 9,8 & 13,3 & 16,7 & 19,2 & 18,8 & 16,3 & 12,1 & 6,8 & 4,0 & 11,0 \\
\hline & in & 0,5 & 1,2 & 2,7 & 5,0 & 8,3 & 11,3 & 13,3 & 13,0 & 10,8 & 7,6 & 3,5 & 1,1 & 6,5 \\
\hline \multirow{3}{*}{ Poitiers } & $T \max$ & 7,2 & 9,1 & 11,8 & 14,9 & 18,5 & 22,2 & 25,1 & 24,6 & 21,9 & 17,2 & 11,0 & 7,8 & 15,9 \\
\hline & Tmoy & 4,2 & 5,4 & 7,3 & 9,8 & 13,3 & 16,6 & 19,1 & 18,6 & 16,3 & 12,4 & 7,3 & 4,7 & 11,3 \\
\hline & $T \min$ & 1,1 & 1,7 & 2,7 & 4,8 & 8,1 & 11,1 & 13,1 & 12,7 & 10,7 & 7,6 & 3,6 & 1,6 & 6,6 \\
\hline
\end{tabular}

mur se démarque particulièrement des autres stations en raison de son été chaud. Cette station enregistre la température maximale moyenne en juillet la plus élevée de tout le Val de Loire $\left(25,6^{\circ} \mathrm{C}\right)$. Ce climat apparemment plus clément que dans les autres stations s'explique par la situation de la station au centre du bassin inférieur de la Loire, et protégée à l'ouest et au sud-ouest par les plateaux et collines les plus élevés de l'espace étudié (les Mauges atteignent $216 \mathrm{~m}$ au Puy des Gardes). Saumur se trouve donc relativement abritée des circulations perturbées d'origine atlantique (Escourrou, 1982 ; Quénol et al., 2008).

11 Ainsi, les nuances climatiques au sein du Val de Loire sont bien présentes et s'expliquent notamment par la distance des stations par rapport à l'océan ou à la Loire, à leur position latitudinale, à la nature des sols et à leur altitude. Le Saumurois en ressort particulièrement privilégié, avec des températures relativement élevées toute l'année par rapport aux autres stations, et avec la deuxième température moyenne annuelle la plus élevée après Nantes, marquant ainsi une rupture dans l'évolution générale vers la continentalité lorsqu'on se dirige vers l'est. Enfin, Romorantin se caractérise par une forte amplitude thermique annuelle et également par de fortes amplitudes thermiques journalières expliquées par sa situation en plaine sableuse. Ces contrastes climatiques régionaux montrent d'une part que les conditions bioclimatiques de la vigne et la qualité du raisin qui en résulte peuvent être différentes d'un vignoble à l'autre; d'autre part, que certaines stations sont plus exposées au gel tardif que d'autres (ex: Romorantin). 


\section{Un réchauffement climatique généralisé}

12 L'observation des températures moyennes annuelles depuis le milieu du $\mathrm{XX}^{\mathrm{e}}$ siècle indique une nette tendance au réchauffement depuis la fin des années 80 pour l'ensemble des stations du Centre-Ouest (figure 2). Le test statistique de Pettitt signale une rupture climatique en 1987 (tableau 4) commune à toutes les stations, à l'exception de Nantes où le changement de tendance a lieu plus tôt, en 1980. La température moyenne annuelle a ainsi augmenté de $0,7^{\circ} \mathrm{C}$ (Poitiers) à $1,2^{\circ} \mathrm{C}$ (Châteauroux et Le Mans) entre les périodes pré-rupture et post-rupture ${ }^{1}$. Même si la rupture de 1987 apparait bien sur la figure 2, nous pouvons également observer un premier maximum de température dans l'après-guerre suivi par un rafraîchissement dans les années 70 : ce sont des oscillations déjà bien connues et décrites (Pédelaborde, 1957 ; Pagney, 1988).

Figure 2 - Evolution des températures (selon une moyenne mobile calculée sur une fenêtre de 5 ans) dans le Val de Loire depuis 1948

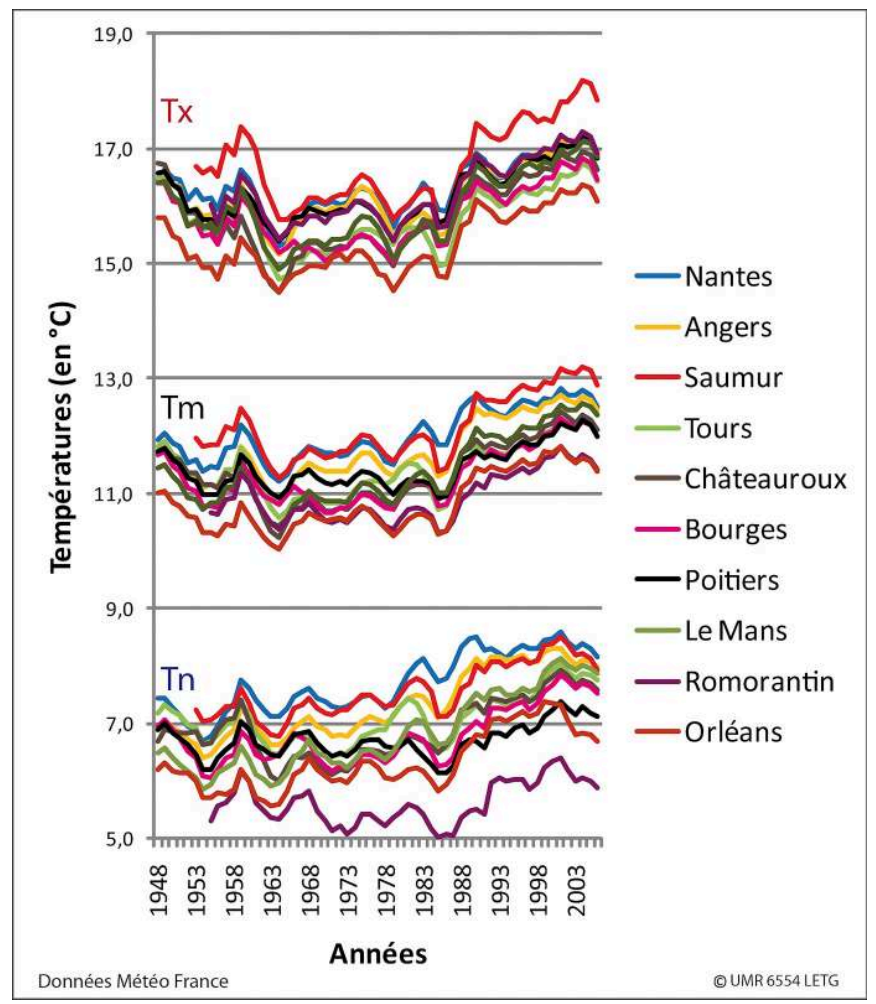

L'observation des températures moyennes maximales (Tx) depuis une soixantaine d'années montre également une nette évolution vers un réchauffement dès la fin des années 80. La rupture de 1987 est stable pour toutes les stations selon le test de Pettitt. Ainsi, la hausse des Tx varie de $0,8^{\circ} \mathrm{C}$ à Nantes à $1,3^{\circ} \mathrm{C}$ à Saumur.

Les nuances des températures moyennes minimales (Tn) sont plus marquées que pour les Tx, et leur évolution depuis la fin des années 50 semble plus contrastée. En effet, la rupture est beaucoup moins stable que pour les Tx, observée de 1980 à 1993 selon les stations. De plus, la courbe d'évolution des Tn laisse présager une stagnation puis une baisse des Tn dans les années 2000 . 
Tableau 4 - Les ruptures climatiques (statistiquement significatives au seuil de $99 \%$ ) dans le Centre-Ouest français (selon le test statistique de Pettitt).

\begin{tabular}{|c|c|c|c|c|c|c|}
\hline \multicolumn{1}{c|}{} & $\begin{array}{c}\text { Rupture } \\
\text { Tm }\end{array}$ & $\begin{array}{c}\text { Hausse } \\
\text { des Tm }\end{array}$ & $\begin{array}{c}\text { Rupture } \\
\text { Tn }\end{array}$ & $\begin{array}{c}\text { Hausse } \\
\text { des Tn }\end{array}$ & $\begin{array}{c}\text { Rupture } \\
\text { Tx }\end{array}$ & $\begin{array}{c}\text { Hausse } \\
\text { des Tx }\end{array}$ \\
\hline Nantes & 1980 & 0,8 & 1980 & 0,9 & 1987 & $\mathbf{0 , 8}$ \\
\hline Angers & 1987 & 1,0 & 1980 & 1,0 & 1987 & 1,0 \\
\hline Saumur & 1987 & 1,0 & 1987 & 0,7 & 1987 & 1,3 \\
\hline Tours & 1987 & 0,9 & 1987 & 0,8 & 1987 & 1,0 \\
\hline Châteauroux & 1987 & 1,2 & 1986 & 1,1 & 1987 & 1,2 \\
\hline Bourges & 1987 & 1,0 & 1987 & 0,9 & 1987 & 1,0 \\
\hline Poitiers & 1987 & $\mathbf{0 , 7}$ & 1993 & $\mathbf{0 , 6}$ & 1987 & 0,9 \\
\hline Le Mans & 1987 & 1,2 & 1980 & 1,2 & 1987 & 1,2 \\
\hline Romorantin & 1987 & 0,8 & 1991 & 0,7 & 1987 & 1,0 \\
\hline Orléans & 1987 & 0,9 & 1987 & 0,8 & 1987 & 1,0 \\
\hline
\end{tabular}

Données : Météo France

En rouge les hausses les plus fortes et en bleu les plus faibles.

Le réchauffement climatique se manifeste de manière généralisée dans tout le Val de Loire et, à une plus large échelle, sur l'ensemble du Centre-Ouest de la France, mais il semble surtout affecter les Tx. Dans tous les cas, cette hausse de température va avoir un impact sur les indices bioclimatiques de la vigne et donc, sur la phénologie des vignobles de Val de Loire.

\section{L'impact du réchauffement climatique sur les indices bioclimatiques}

Certains indices bioclimatiques sont de très bons indicateurs des variations phénologiques de la vigne et permettent de comprendre les relations entre le climat et la plante. Ils se basent la plupart du temps sur des sommes de températures, élément qui explique pourquoi cette étude retient l'évolution des degrés jours de Winkler et l'indice de Huglin.

\section{Les degrés jours de Winkler}

L'indice des degrés jours de Winkler (Winkler et al., 1974) se calcule sur la saison phénologique de la vigne (avril à octobre) et permet de connaître les besoins en chaleur de la plante pour le développement des différentes phénophases (débourrement, floraison, véraison et maturité). Il est donc souvent utilisé dans des travaux étudiant l'impact du climat sur la vigne. L'étude est menée ici entre d'une part la période calculée avant la rupture d'homogénéité climatique des températures moyennes annuelles, et d'autre part la période située après la rupture (figure 3).

Une hausse de l'indice est constatée pour toutes les stations, répercussion de la hausse des températures moyennes. Cependant, nous avons vu que la hausse des températures n'est pas la même partout, contrastes logiquement retrouvés avec l'indice stationnel. Nantes connaissant l'une des hausses des températures moyennes annuelles parmi les plus faibles, la hausse de l'indice est donc plus modérée que dans les autres villes $(+148$ Dj). En revanche, la hausse de l'indice est maximale dans l'Anjou (+198 Dj) et surtout le Saumurois $(+233 \mathrm{Dj})$. Ainsi, Saumur qui connaît un climat plus doux que ses voisines, notamment du fait de sa position géographique, se réchauffe également plus vite. Tours a également gagné $184 \mathrm{Dj}$ entre les deux périodes. 
Figure 3 - Evolution de l'indice des degrés jours avant et après la rupture d'homogénéité des séries climatiques observée dans le Val de Loire

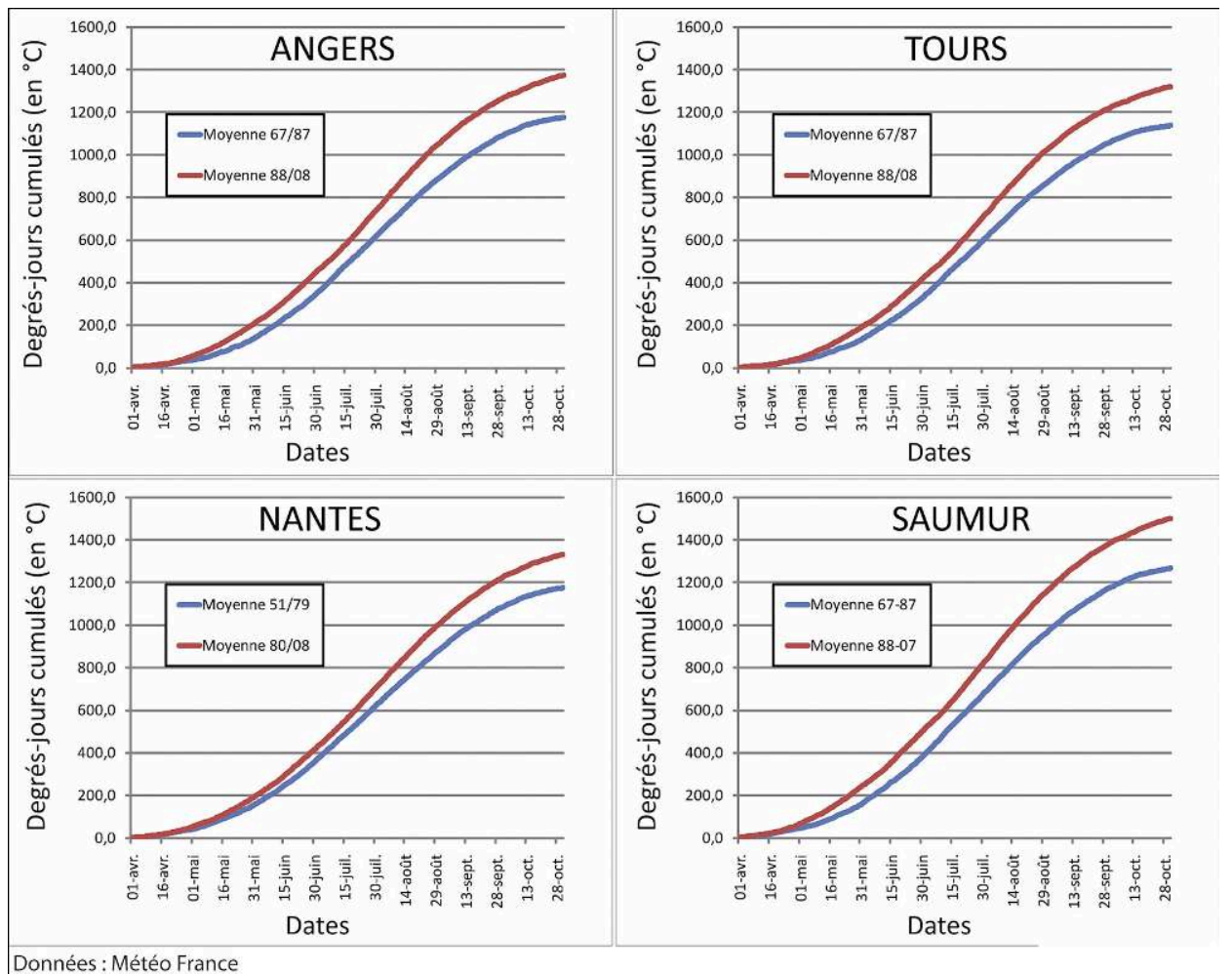

Cette hausse de l'indice des degrés jours de Winkler a donc des conséquences sur la phénologie de la vigne. L'apport de chaleur pour chaque phénophase étant atteint plus tôt dans la saison, les viticulteurs observent déjà une plus grande précocité de ces stades, parfois de 15 jours à 3 semaines, comme cela a pu être montré en Bourgogne ou dans le Bordelais (Jones et al., 2005 ; Madelin et al., 2008). De plus, la croissance de la vigne et, en particulier, la maturité, se déroule désormais sous des conditions plus chaudes, notamment au mois d'Août. Le test de Pettitt nous indique pour les Tx du mois d'Août une rupture climatique en 1986 à Saumur (significativité : 99\%) et Nantes (95\%) et en 1988 à Angers (99\%) et Tours (95\%). Ainsi les Tx moyennes entre la période pré- et post-rupture ont augmenté d'environ $2^{\circ} \mathrm{C}$ selon les stations, passant de $24,6^{\circ} \mathrm{C}$ à $26,8^{\circ} \mathrm{C}$ à Saumur, de $23,7^{\circ} \mathrm{C}$ à $25,3^{\circ} \mathrm{C}$ à Nantes, de $24,2^{\circ} \mathrm{C}$ à $26^{\circ} \mathrm{C}$ à Angers et de $24,3^{\circ} \mathrm{C}$ à $26^{\circ} \mathrm{C}$ à Tours. Ces changements bioclimatiques ont des impacts sur les caractéristiques du raisin récolté, notamment la quantité de sucre et l'acidité et donc, sur la typicité des vins produits.

\section{L'indice de Huglin}

Un autre indice utilisé en viticulture permet de classer les climats par catégorie allant du plus froid au plus chaud. Il s'agit de l'indice héliothermique de Huglin (IH), qui a ensuite été développé à l'échelle de la Californie. L'évolution de cet indice est étudiée ici sur la plus longue période possible et pour les mêmes stations que pour le précédent indice des degrés jours (figure 4), afin de vérifier si la valeur de IH a également augmenté depuis le milieu du $\mathrm{XX}^{\mathrm{e}}$ siècle et donc, si les caractéristiques des climats viticoles du Val de Loire ont changé. 
Figure 4 - Evolution temporelle de l'indice héliothermique de Huglin dans le Val de Loire.

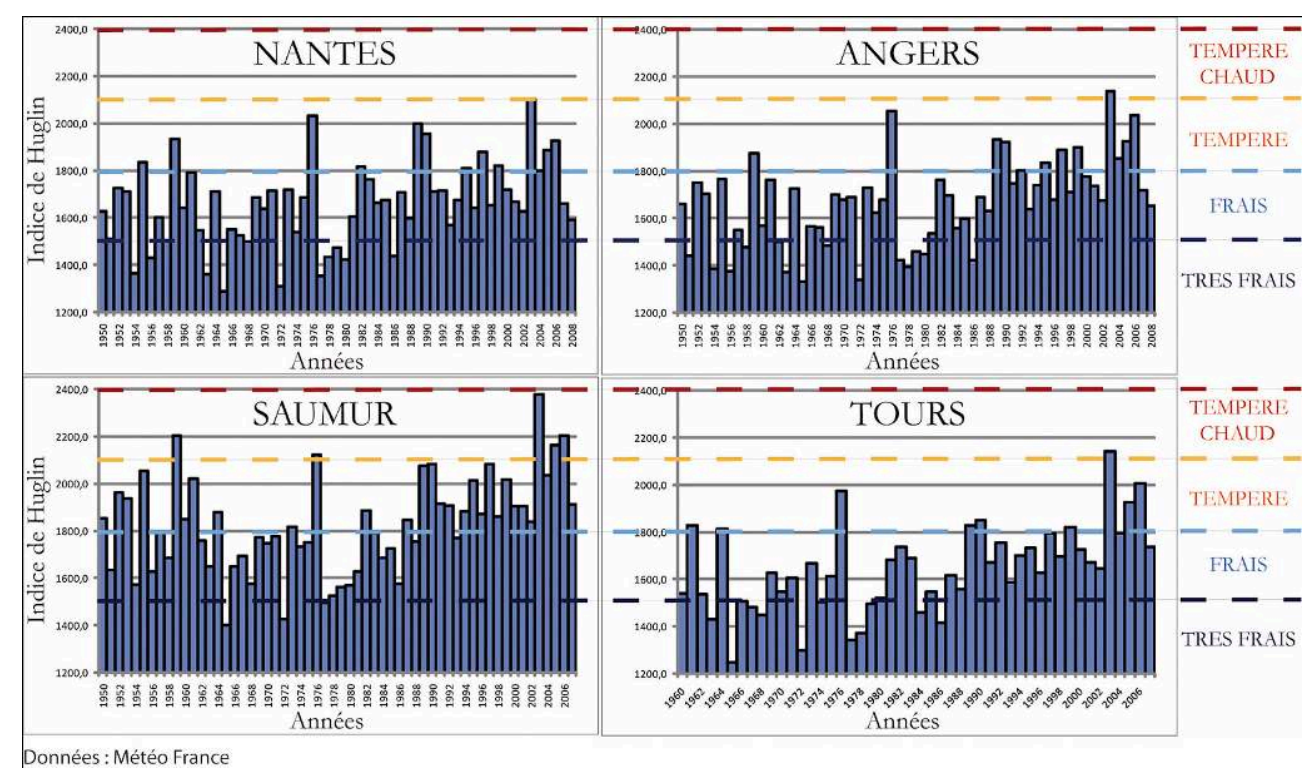

Le réchauffement climatique se répercute sur l'indice de Huglin avec une évolution vers des valeurs plus élevées à partir des années 80 . Nous retrouvons la période chaude des années 50 et la période plus froide des années 70, mais la hausse des valeurs de $\mathrm{IH}$ est plus sensible dès la fin des années 80 . Le test statistique de Pettitt confirme la rupture climatique pour cet indice en 1981 pour Nantes, 1986 pour Saumur et 1988 pour Angers et Tours. Ainsi, la plupart des stations étaient classées, avant la rupture climatique, dans la catégorie des climats viticoles frais voire en climat tempéré pour Saumur dans les années 50 et au début des années 60 . Après la rupture d'homogénéité sérielle, les stations se trouvent plutôt classées dans des climats désormais plus tempérés voire tempérés chauds à Saumur au début des années 2000. Cependant, Tours qui est au départ une station plus fraîche que les autres, n'est passée dans la catégorie des climats tempérés qu'au début des années 2000 grâce à une succession d'années chaudes.

Nous pouvons donc constater une hausse de l'indice de Huglin dans le Val de Loire depuis le milieu du XXe siècle. Si nous observons le profil de l'indice à toutes les stations, Saumur semble plus sensible aux périodes chaudes. En effet, la variabilité temporelle semble plus marquée en amplitude que dans les autres stations, d'où sans doute la réaction plus prononcée de Saumur au réchauffement climatique contemporain. Cette hypothèse reste cependant à confirmer. Cette étude est intéressante, car elle permet de poser la question de l'évolution future de cet indice. Chaque catégorie de climat correspond à des types de cépages pouvant être plantés afin d'optimiser la récolte. Si les vignobles changent de catégories climatiques, c'est l'ensemble des cépages qui pourrait être remis en question.

\section{Conclusion}

A l'échelle régionale, les nuances climatiques entre des stations peuvent être marquées. En effet, nous avons vu qu'à l'échelle du Centre-Ouest français et, en particulier, du Val de Loire, ces nuances existent et sont dues en partie à la distance des stations à l'océan, 
à la topographie et à leur position latitudinale. Ainsi, Saumur, de par sa position d'abri, ressort comme la station la plus chaude de toutes les stations alors que Romorantin, de par sa position dans une "vallée " au sol sableux connait des amplitudes thermiques relativement élevées. Ces contrastes des conditions climatiques viticoles expliquent, en partie, la diversité des vins produits dans cette région. Le réchauffement climatique contemporain vient modifier ces caractéristiques agro climatiques. Le changement de tendance dans les séries de températures apparaît à la fin des années 80 pour les températures maximales et moyennes (hormis pour Nantes). L'évolution des températures minimales est plus complexe à analyser et mérite des travaux plus poussés. La rupture climatique de Nantes en 1980 doit également être analysée plus précisément afin de chercher si cette discontinuité peut être d'origine instrumentale ou bien si cette rupture se retrouve dans des stations voisines à climat maritime, comme Saint-Nazaire par exemple, ce qui serait alors une particularité de la Loire Atlantique.

La hausse des températures se répercute sur les indices bioclimatiques viticoles. Ainsi, les degrés jours ont augmenté de plus de $230 \mathrm{Dj}$ à Saumur entre les périodes moyennes observées avant et après la rupture climatique. L'indice de Huglin place désormais les vignobles du Val de Loire dans des climats plus doux (tempéré à tempéré chaud). Ces changements de caractéristiques des climats viticoles peuvent expliquer une plus grande précocité saisonnière du développement de certains stades phénologiques de la vigne dans le Val de Loire. Une autre étape de cette étude sera de descendre à des échelles spatiales plus fines, nécessaires pour une étude sur les vignobles, puisque les nuances climatiques sont également très marquées aux échelles topoclimatiques et locales. Pour ce travail, un réseau de stations météorologiques complètes de type Campbell a été installé en 2008 dans l'Anjou et le Saumurois afin de compléter le réseau de Météo - France. De même, des capteurs déportés de température (type Tinytag) ont été installés à partir de l'année 2008 dans les coteaux du Layon avec une densité suffisamment importante pour caractériser certaines différences climatiques à l'échelle d'une parcelle et préciser ainsi les conditions topoclimatiques particulières qui permettent la production des vins liquoreux.

\section{BIBLIOGRAPHIE}

Avila F., 1986. Janvier 1985 et les grands hivers du passé dans les Landes et le Sud-Ouest. Projet d'Action Educative, Lycée G. Crampe, Aire-sur-l'Adour, 155 p.

Escourrou G., 1981. Climat et Environnement. Les facteurs locaux du climat. Masson, Paris, 184 p.

ESCOURROU G., 1982. Le climat de la France. PUF, coll. Que Sais-je ?, 126 p.

OkeT.R., 1978. Boundary Layer Climates. Methuen \& Co. Ltd., London, 372 p.

Galet P., 2000. Précis de viticulture, éditions Galet, 602 p.

Huglin P. et SCHNEIDER C., 1998. Biologie et écologie de la vigne, Paris, Lavoisier, 370 p. 
Jones G., Duchene E., Tomasi D., Yuste J., Braslavska, O., Schultz H., Martinez C., Boso S., Langellier F., Perruchot C. et Guimberteau G., 2005. Changes in 53 european winegrape phenology and relationships with climate, GESCO 2005, Allemagne, Vol. 1, p. 55-62.

Lobell D.-L., Field C.-B, Cahill K.-N. et Bonfils C., 2006. Impacts of future climate change on California perennial crop yields: Model projections with climate and crop uncertainties. Agricultural and Forest Meteorology n¹41, p. 208-218.

Lubes H., Masson .J.M., Servat E., Paturel J. B., Kouame B. et Boyer J. F., 1994. Caractérisation de fluctuations dans une série chronologique par applications de tests statistiques. Etude bibliographique. UR2-DEC Orstom, Montpellier. $115 \mathrm{p}$.

Madelin M., Perard J. et Chabin J.-P., 2007. Les conséquences du réchauffement climatique sur les vignobles de Côte-d'Or. Revue des Enologues, 125 HS, p. 12-13.

Madelin M., Chabin J.-P., Bonnefoy C., 2008. Global Warming and its consequences on the Beaune vineyards. Enometrica, p 9-19.

Pagney P., 1988. Climats et cours d'eau de France. Masson, Paris, 166 p.

Pedelaborde P., 1957. Le climat du Bassin Parisien. Génin, Paris, 539 p.

Pettitt A.-N, 1979. A non-parametric approach to the change-point problem. Applied Statistics 28, $n^{\circ} 2$, p. 126.

Pinty J.P., Mascart P., Richard E. et Rosset R., 1989. An investigation of mesoscale flows induced by vegetation inhomogeneities using an evapotranspiration model calibrated against HAPEXMOBILHY data. J.l of Applied Meteor. 28, p. 976-992.

Planchon O., 1994. À propos de la notion de climat maritime. Études Méditerranéennes, 16, p. $119-130$.

Planchon O., 1997. Les Climats Maritimes dans le Monde. Presses Universitaires du Septentrion, Villeneuve d'Ascq, $233 \mathrm{p}$.

Quenol H., Planchon O. et Wahl, L., 2008. Méthodes d'identification des climats viticoles. Bulletin de la Société Géographique de Liège, 51, p. 127-137.

Seguin B., 2010. Coup de chaud sur l'agriculture. Ed. Delachaux et Niestlé, 205 p.

Vaudour E., 2003. Les terroirs viticoles : définitions, caractérisation et protection. Ed. Dunod, 293 p.

Webb L.B., Whetton P.H. et Barlow (Snow) E.W.R, 2007. Future Climate Change Impacts on Australian Viticulture. Actes du colloque international à Dijon : « Réchauffement climatique, quels impacts probables sur les vignobles?», $10 \mathrm{p}$.

White M.A., Diffenbaugh N.S., Jones G.V, Pal J.S. et Giorgi F., 2006. Extreme heat reduces and shifts United States premium wine production in the 21st century. Environmental Sciences, vol. $103, n^{\circ} 30,6 \mathrm{p}$.

Winkler A.J., Cook J.A., Kliewer W.M. et Lider L.A., 1974. General viticulture, Berkeley, University of California, 710 p.

\section{NOTES}

1. La période post-rupture contient le même nombre d'années que la période pré-rupture. 


\section{RÉSUMÉS}

La variabilité spatiale et temporelle du climat à l'échelle régionale peut parfois être marquée. Les différences spatiales observées peuvent être dues à la proximité ou non d'un océan, à la topographie, l'altitude, ou bien encore à la position latitudinale des stations. Le Val de Loire, l'une des grandes régions viticoles françaises, du fait de son orientation est-ouest, connaît des nuances climatiques assez marquées avec une influence océanique à l'ouest, liée à la proximité de l'Océan Atlantique, qui s'atténue en allant vers l'est où le climat est marqué par des caractéristiques plus continentales. De ce fait, différentes caractéristiques climatiques viticoles sont observées dans la vallée de Loire. Depuis le milieu du $\mathrm{XX}^{\mathrm{e}}$ siècle, un réchauffement généralisé est observé à partir de toutes les stations Météo - France. Ce réchauffement est plus sensible depuis la fin des années 80 . Cette augmentation de température se répercute logiquement sur les indices bioclimatiques viticoles.

Spatial and temporal climate variability at regional scale can sometimes be important. Spatial observed differences can be explained by the nearness or not of ocean, topography, elevation or latitude... Important climatic nuances are observed in the Loire Valley, one of the big French wine-producing areas, because of its East-West orientation. The oceanic influences close to the coast are gradually deteriorated to the East with more continental influences. As a result various climatic characteristics are observed in the Loire Valley vineyards. Since the middle of the $20^{\text {th }}$ century, a regional warming has been noticed. This warming has been more significant since the end of the 80 's. This increase of temperature is reflected in the bioclimatic indices.

INDEX

Keywords : bioclimatic indices, Loire Valley, regional scale, temperature, warming

Mots-clés : indice bioclimatique, réchauffement, température, Val de Loire

\section{AUTEURS}

\section{CYRIL BONNEFOY}

Cyril Bonnefoy est membre du Laboratoire COSTEL, UMR 6554 LETG du CNRS, Université Rennes2

- Haute Bretagne. Cyril.bonnefoy@univ-rennes2.fr

\section{HERVÉ QUENOL}

Hervé Quenol est membre du Laboratoire COSTEL, UMR 6554 LETG du CNRS, Université Rennes2 Haute Bretagne. Herve.quenol@uhb.fr

\section{OLIVIER PLANCHON}

Olivier planchon est membre du Laboratoire COSTEL, UMR 6554 LETG du CNRS, Université Rennes2 - Haute Bretagne. Olivier.planchon@uhb.fr

\section{GÉRARD BARBEAU}

Gérard Barbeau est membre de l'INRA UE 1117, UMT Vinitera, Beaucouzé.

Gerard.barbeau@angers.inra.fr 